\title{
Malaria parasitaemia among long distance truck drivers in the Niger delta of Nigeria
}

\author{
*Erhabor $\mathrm{O}^{1}$, Azuonwu $\mathrm{O}^{2}$, Frank-Peterside $\mathrm{N}^{3}$
}

1. Department of Medical Laboratory Sciences, Niger Delta University Ammasoma Bayelsa State

2. Department of Medical Laboratory Sciences, Rivers State University of Science and Technology, Port Harcourt

3. Department of Microbiology, University of Port Harcourt

\begin{abstract}
Background: Malaria is the leading cause of morbidity and mortality in sub-Saharan Africa.

Objective: The aim of this study was to determine the prevalence of malaria among long distance truck drivers in the Niger Delta of Nigeria.

Methods: A total of one hundred consecutively recruited long distance truck drivers aged 21-60 years, with a mean age of $42.36 \pm 5.23$ years were screened for the presence of malaria parasitaemia.

Results: Out of the 100 truck drivers screened, 35 (35\%) were positive for malaria while 65 (65\%) were negative. Plasmodium falciparum was responsible for all cases of malaria infection. The highest prevalence of malaria occurred among drivers in the 51-60 years age group (40.5\%). The mean and standard deviation (SD) of parasite load was 1020 (125) parasites/il in subjects positive for malaria. The mean CD4 count wassignificantly higher among non-parasitized truck drivers compared to P. falciparum parasitized drivers $820 \pm 42.0$ (731-902 cells / $\boldsymbol{\mu l}$ ) and $570 \pm 30.0$ (510-630 cells/ $\boldsymbol{\mu l}$ ) respectively (chi square $=74.00 ; \mathrm{p}=0.03)$. We observed a significant negative correlation between plasmodial infection and CD4 lymphocyte count among Plasmodium falciparum-infected subjects with $\mathrm{r}=-0.56(\mathrm{p}=0.001)$.

Conclusion: Preventative strategies including regular chemoprophylaxis, intermittent preventive treatment with antimalarials and provision of insecticide-treated bed nets should be implemented.
\end{abstract}

Keywords: CD4 count, Malaria, Long distance drivers, Niger Delta, Nigeria

African Health Sciences 2012; 12(2): 98 - 103 http://dx.doi.org/10.4314/ahs.v12i2.4

\section{Introduction}

Malaria poses an enormous public health burden and greater than $75 \%$ of the global clinical episodes of malaria infection each year are concentrated in Africa. Malaria is a life-threatening disease caused by the Plasmodium parasite, which is transmitted to people through the bites of infected mosquitoes. According to latest estimates from the World Health Organization (WHO), in 2009, there were 225 million cases of malaria and an estimated 781, 000 deaths worldwide. Most of these deaths occur among children living in the WHO African Region (mainly sub-Saharan Africa) ${ }^{1}$. Over the years, however, the global malaria prevalence has gone down thanks to the WHO control interventions. Malaria has infected humans for over 50, 000 years, and Plasmodium

\section{*Correspondence author: \\ Dr Erhabor Osaro \\ Blood Sciences Department \\ Bolton Hospital Foundation NHS Trust \\ Minerva Road Bolton UK BL4 0JR \\ Telephone: +447932363217 \\ Email: n_osaro@yahoo.com}

may have been a human pathogen for the entire history of the specie ${ }^{2,3}$. Malaria is a major cause of low productivity and poverty and slows economic growth by up to $1.3 \%$ per year in endemic countries ${ }^{4}$. It accounts for $40 \%$ of public health expenditure and is estimated to cost Africa more than US\$ 12 billion every year in lost Gross Domestic Product $(\mathrm{GDP})^{5}$. Resistance of Plasmodium falciparum parasites to ntimalarial drugs has fuelled an increase in malaria prevalence and malaria specific mortality during the past decade in sub-Saharan Africa. Promising efficacy results from field trials of Artemisinin containing Combination Therapy (ACT) are a source of optimism and numerous African countries have changed their first line malaria drug policy to ACT in order to counter high levels of resistance ${ }^{6,7}$.

There is paucity of data on the prevalence of malaria infection among migratory long distance truck drivers in the Niger Delta of Nigeria. The aim of this study was to investigate the prevalence of malaria this group of people. 


\section{Methods}

\section{Study area}

The study was carried out among long-distance truckers plying one of the major national highways in the Niger Delta of Nigeria. Major halt points of truckers were identified and those halting at these Eagle Cement Trucks Park, Iwofe, Onne Trucks Park, and Eleme were studied. Written informed consent was obtained from each subject. The Niger Delta of Nigeria is said to be the world's largest wetland. This 36, $000 \mathrm{sq} \mathrm{km} \mathrm{(14,} 000$ square miles) of marshland, creeks, tributaries, and lagoons drain the Niger River into the Atlantic at the Bight of Biafra. Transportation through this ecosystem is usually via rivers and creeks that snake through dense mosquitoinfested swamps. There is a high incidence of malaria in the area. Indeed, early European visitors to the area described it as the 'white man's grave-yard' because of the high malaria-related mortality rate they experienced.

\section{Sample collection}

Four milliliters of whole venous blood was collected from the anticubital vein of each study subject into EDTA anticoagulated tube. The EDTA sample was used for the determination of CD4 lymphocyte count and malaria parasite investigation. Testing was carried out in the diagnostic/research laboratory at the Department of Medical Laboratory Science, Rivers State University of Science and Technology and the Pathology Department of Braithwaite Memorial Hospital, both in Port Harcourt, Nigeria. All test procedures were carried out according to the manufacturers' instructions and following the standard operating procedures for the investigations.

\section{Study population}

Subjects for this cross-sectional study included a total of 100 consecutively recruited long - distance drivers in Port Harcourt Rivers State, the heart of the Niger Delta of Nigeria. The mean age and age range of drivers was $42.36 \pm 5.23$ and 21 to 60 years respectively. Inclusion criteria were; age between 21 to 60 years, more than a year in the truck-driving occupation, and willingness to offer written informed consent to participate in the study after counseling. All subjects were offered pre and postmalaria test counseling. Ethical approval was sought for and obtained from both the management of the Truck Drivers Association and the Ethics Committee of the Medical Laboratory Science Department of the Rivers State University of Science and Technology where the study was carried out. Demographic data such as age and gender of truck drivers were collected via an intervieweradministered questionnaire. The study was carried out during the rainy season between April and June 2009.

\section{Malaria diagnosis}

Malaria parasitemia was defined by the presence of asexual forms of Plasmodium species on quantitative buffy coat (QBC) malaria test and confirmed by microscopic examination of the peripheral blood in all subjects. Malaria testing was done using the QBC Autoread TM Plus centrifugal Haematology and Malaria analyzer (Model 428576, 2006; QBC Diagnostics Inc, Philipsburg, PA, USA). Fifty-five microliters of blood is taken in a $\mathrm{QBC}^{\mathrm{TM}}$ tube coated with Acridine Orange (AO) fluorescent dye and centrifuged for five minutes. The parasites in a QBC tube, when viewed with the Paralens microscope adaptor, are seen concentrated in the area below the Buffy Coat, fluorescing in the background of dark Red Blood Cells (RBC). Blood smears (thin and thick films) were prepared for all QBC malaria-positive samples and stained with Field's stain (for confirmation, speciation, and parasite load determination) and were read for 200 fields. Parasite counts were reported per 500 White Blood Cells (WBC) and for counts above 1,000 parasites per 500. Malaria parasitaemia was defined by the presence of asexual forms of P. falciparum on QBC malaria test and confirmed by microscopic examination of the peripheral blood in truck drivers with or without elevation of temperature (aural < $37.5^{\circ} \mathrm{C}$ ), history of fever, or any of the following symptoms: headache, dizziness, joint pain, anorexia, and nausea.

\section{CD4 lymphocyte count}

The CD4 lymphocyte count was determined using the Partec Cyflow counter (Phywe AG, Gottingen, Germany), using Ethylenediamine Tetra Acetic acid (EDTA) anticoagulated sample. The $\mathrm{CyFlow}{ }^{\circledR}$ Counter is a fully equipped portable and ultra compact desktop flow cytometer dedicated for routine CD4 and CD4\% counting (as well as total lymphocyte and WBC counting).

\section{Statistical analysis}

Data were entered and analyzed using statistical package SPSS version 9 (SPSS Inc., Chicago, IL). Statistical analyses included descriptive analysis of 
mean, standard deviation, and Chi-square analysis. A p- value of $\geq 0.05$ was considered to be statistically significant in all statistical analyses. Correlation was compared using linear regression analysis.

\section{Result}

The results obtained showed that out of the total number of 100 truck drivers aged 21-60 years ( mean $42.36 \pm 5.23$ years) screened, $35(35 \%)$ were positive for malaria while $65(65 \%)$ were negative. Plasmodium falciparum was responsible for all malaria infection observed among truck drivers. The highest prevalence of malaria occurred among drivers in the 51-60 years age group (40.5\%). The the lowest prevalence occurred in the 31 - 40 years age groups. Figure 1 show the distribution of malaria infection among truck drivers based on age groups. The mean and standard deviation (SD) of parasite load in subjects positive for malaria was 1020 (125) parasites/andl. The mean values, standard deviation (SD) and ranges of the CD4 count were significantly higher among non-parasitized truck drivers compared to Plasmodium falciparum parasitized drivers 820 (42.0) (731-902 cells/ $\boldsymbol{\mu l}$ ) and 570(30.0) and $(510-630$ cells $/$ il) respectively (chi square $2=$ 74.00; $\mathrm{p}=0.03$ ). We observed a significant negative correlation between plasmodial infection and CD4 lymphocyte count among Plasmodium malaria infected subjects, with $\mathrm{r}=-0.56(\mathrm{p}=.001)$. Table 1 shows the mean values of $\mathrm{CD} 4$, parasite load, and age of subjects

Figure 1: Distribution of malaria among truck drivers based on age groups

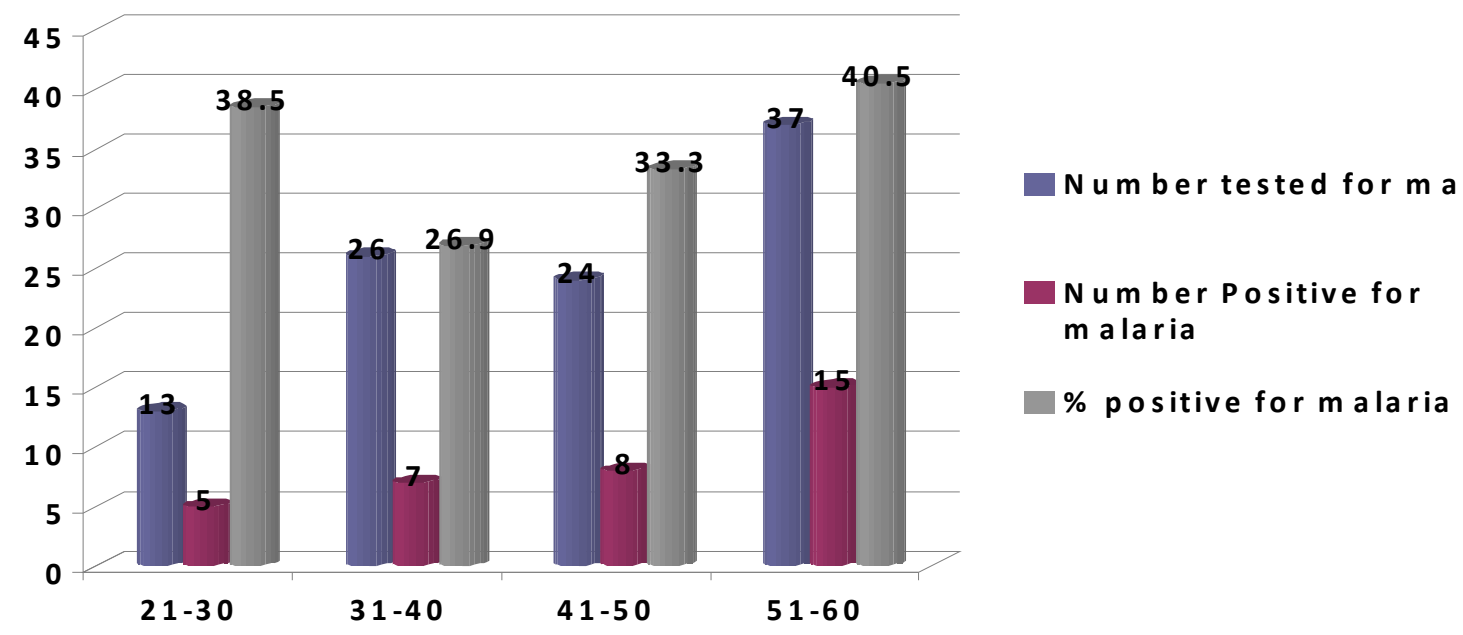

Table 1: Mean CD4, parasite load and age of Truck drivers

\begin{tabular}{lllll}
\hline $\begin{array}{l}\text { Variable } \\
(\text { mean } \pm \text { sd })\end{array}$ & $\begin{array}{l}\text { Malaria positive } \\
\text { drivers }\end{array}$ & $\begin{array}{l}\text { Malaria negative } \\
\text { drivers }\end{array}$ & chi square & P-value \\
\hline CD4 countcells $/ \mu 1$ & $570 \pm 30^{a}$ & $820 \pm 42$ & 74.00 & 0.03 \\
\hline
\end{tabular}

a Significant difference observed between malaria-infected and non-infected pregnant women $(\phi=0.01)$

\section{Discussion}

Plasmodium falciparum has a leading role in the causation of malaria in West Africa. In this study carried out in the malaria endemic Niger Delta of Nigeria, we recorded an overall prevalence of $35 \%$ malaria infection among long- distance drivers studied. Plasmodium falciparum was found to be the predominant specie. Furthermore, the highest prevalence of malaria occurred in the 51-60 years age group. Researchers from different parts of the country have reported a varying prevalence of malaria among pregnant women, ranging from $29 \%$ in south East Nigeria ${ }^{8}, 31 \%$ in Abuja ${ }^{9}$, and $39.2 \%$ in Kano to $60 \%$ in Lagos ${ }^{10}$. Similarly, another study ${ }^{11}$ among Antenatal clients (ANCs) from a peri-urban community in Lagos reported a $44.2 \%$ prevalence of asymptomatic malaria at booking among primgravidae attending a Primary Health Care (PHC) facility, which was significantly lower than 
$33.6 \%$ prevalence found among multigravidae ${ }^{12}$. A case control study in Nnewi, Nigeria among pregnant and non pregnant women observed a prevalence of parasitaemia of $79.3 \%$ among pregnant women and $31.5 \%$ among non pregnant controls ${ }^{13}$. Similarly, a study among 240 apparently healthy children in a cross sectional descriptive study in the Niger Delta of Nigeria observed a prevalence of Plasmodium falciparum malaria of $27.5 \%{ }^{14}$. A population based study in Oshogbo metropolis, southwest, Nigeria to investigate the prevalence pattern and clinical presentation of falciparum malaria over a period of 12 months (August 2004 toJuly 2005) observed a prevalence of Plasmodium falciparum of $52.8 \%$ 15.

There are several reasons for the high prevalence of malaria observed among migratory long- distance drivers. One of the factors contributing to the re-emergence of malaria is human migration. People move for a number of reasons, including environmental deterioration, economic necessity, occupation, conflicts, and natural disasters. These factors are most likely to affect the poor, many of whom live in or near malarious areas. Identifying and understanding the influence of these population movements can improve prevention measures and malaria control programs ${ }^{16}$. Movements of people can contribute to the transmission of malaria infections.

In addition, programmes for malaria control/eradication, and for the improvement of public health in general, are hindered when applied to populations which are in whole or in part mobile. Historically, population movement has contributed to the spread of diseases ${ }^{17}$. Various malariaeradication campaigns failed due to nonconsideration of this factor in the 1950s and $1960 \mathrm{~s}^{18}$. Movement of infected people from endemic areas to areas where the disease had been eradicated led to its resurgence. Population movement is also increasingly implicated in the spread of drug resistance in malaria ${ }^{19}$. Poor housing and sanitation, lack of proper drainage of surface water, and use of unprotected water reservoirs that increase human vector contact and vector breeding are prevalent in the Niger delta of Nigeria. These factors increase vulnerability to malaria infection particularly among truck drivers because of the migrational nature of their job. Truck drivers are also vulnerable to malaria infection in the makeshift temporary shanty towns in which they halt to have a drink, smoke, and interact with commercial sex workers. These makeshift environments provide habitats suitable for mosquito breeding.

More so, most of these remote communities where truck drivers halt along the trucking routes have only limited access to health services. This delays the possible access to malaria treatment among long distance drivers. Truck drivers spend limited time with their families because of their frequent cross border movements, and due to their restricted financial situation, they may not be able to afford accommodation. Most times they are exposed to mosquitoes because they stay mostly on the road without access to insecticide-treated nets and are often bitten by female anopheles mosquitoes ${ }^{20}$. Indoor spraying with insecticides for malaria control has proved ineffective particularly among longdistance drivers as most mosquito bites occur outdoors. Previous reports indicate that indoor residual spray (IRS) with insecticides and insecticidetreated bed nets (ITNs) are the most important malaria vector control tools in the tropical world ${ }^{21}$. Application of both tools in the same locations is being implemented for malaria control in endemic and epidemic Africa ${ }^{22}$.

The reason for the high prevalence observed in this study may be due to the fact that the study was carried out in the rainy season. Previous studies indicate that infections increase during the rainy season owing to better availability of water for breeding sites for female mosquitoes ${ }^{22-23}$. The majority of the truck drivers studied were asymptomatic although some of them complained of regular fatigue, aches and weakness. This observation is consistent with a previous report which demonstrated that a history of previous clinical malaria significantly reduces the risk of WHO-defined severe falciparum malaria ${ }^{24}$

There are several reasons why asymptomatic malaria may persist among truck drivers. These include hard working conditions which involve daily risk-taking behaviors, and long separation from spouses. Work in remote and poor mosquitoinfested environments is characterized by limited access to convenient health services, coupled with truck drivers preference to use self medication, quacks, and traditional healers for the treatment of malaria making them more vulnerable to having a residual malaria infection due to suboptimal use of antimalarials and possible development of drug resistant malaria ${ }^{25}$. One key strategic intervention is provision of early diagnosis and prompt effective treatment. A major setback in most settings in sub- 
Saharan Africa has been the development of drug resistance to commonly used antimalarials. Resistance of Plasmodium falciparum parasites to antimalarial drugs has fuelled an increase in malaria prevalence and malaria specific mortality during the past decade in sub-Saharan Africa. Promising efficacy results from field trials of ACT are a source of optimism and numerous African countries have changed their first line malaria drug policy to ACT to counter high levels of resistance ${ }^{5-6}$.

We observed a significantly lower CD4 count among Plasmodium falciparum- infected truck drivers compared to those uninfected. We observed a significant negative correlation between malaria infection and CD4 lymphocyte count. Malaria seems to have a negative impact on the CD4 lymphocyte count. Previous reports indicated that persons with severe malaria were more likely to have a CD4 count $<350 / \mu \mathrm{L}$ than asymptomatic controls ${ }^{26}$.

We observed that the predominant plasmodium specie responsible for malaria infection among truck drivers in the Niger Delta of Nigeria was Plasmodium falciparum. This finding is consistent with previous reports ${ }^{27-29}$ in the region which found Plasmodium falciparum as the predominant specie responsible for malaria in Nigeria. Mapping the global distribution of malaria motivated by a need to define populations at risk for appropriate resource allocation, and to provide a robust framework for evaluating its global economic impact, has shown that malaria infection particularly caused by Plasmodium falciparum has been geographically restricted and remains entrenched in poor areas of the world particularly in sub Saharan Africa ${ }^{30}$.

\section{Conclusions}

This study indicates that long distance drivers are susceptible to malaria infection. There is a need for interventions such as mass media campaigns, peer / outreach education, and life skill programmes in the halting point where these drivers meet in order to bring about a reduction in the prevalence of malaria infection. There is also the need for the promotion of insecticide-treated bed nets (ITNs), intermittent preventive treatment (IPTp), and effective case management of malarial illness among long distance drivers.

\section{References}

1. World Health Organization .World Malaria Report. Geneva: World Health Organization. 2010.

2. Joy DA, Feng X, Mu J, Furuya T, Chotivanich $\mathrm{K}$, Krettli AU, et al. Early origin and recent expansion of Plasmodium falciparum. Science. 2003 Apr 11; 300(5617):318-21.

3. Cox F. History of human parasitology. Clinical Microbiology Review 2002; 15 (4): 595-612.

4. Sachs J, Malaney P. The economic and social burden of malaria. Nature 2002; 415:680-85.

5. Roll Back Malaria. Malaria in Africa. Accessed from RBM website May 2007: http:// www.rbm.who.int/cmc_upload/0/000/015/ 370/RBMInfosheet_3.htm

6. Njuguna J, Qader SS. Challenges associated with the scaling up of Artemisinin Combination Therapy in sub-Saharan Africa. A Review Article. Libyan Journal of Medicine 2008; 3(1): 42-48.

7. Adjuik M, Babiker A, Garner P, et al. Artesunate combinations for treatment of malaria: meta analysis. Lancet 2004; 363:9-17.

8. Nwonwu EU, Ibekwe PC, Ugwu JI, Obarezi HC, Nwagbara OC. Prevalence of malaria parasitaemia and malaria related anaemia among pregnant women in Abakaliki, South East Nigeria. Niger J Clin Pract. 2009 Jun; 12(2):182-186.

9. Agboghoroma OC, Elegba OY, Ladipo OP, Umezulike AC, Efetie RE, Tabansi S. Prevalence of Asymptomatic Malaria Parasitaemia in Pregnant Women at First Antenatal Visit in Abuja. Trop J Obstet Gynaecol 2004; 21:S36.

10. Gajida AU, Iliyasu Z, Zoakah AI. Malaria among antenatal clients attending primary health care facilities in Kano state, Nigeria. Ann Afr Med. 2010 Jul-Sep; 9(3):188-193.

11. Okwa OO. The status of malaria among pregnant women: A study in Lagos, Nigeria. Afr J Reprod Health 2003; 7:77-83.

12. Anorlu RI, Odum CU, Essien EE. Asymptomatic malaria parasitaemia in pregnant women at booking in a primary health care facility in a periurban community in Lagos, Nigeria. Afr J Med Med Sci 2001; 30:39-41.

13. Nnaji GA, Ikechebelu JI, Okafor CI. A comparison of the prevalence of malaria parasitaemia in pregnant and non pregnant women. Niger J Med. 2009; 18(1):47-51.

14.Jeremiah ZA, Jeremiah TA, Emelike FO. Frequencies of some human genetic markers and their association with Plasmodium falciparum 
malaria in the Niger Delta, Nigeria. J Vector Borne Dis. 2010 Mar; 47(1):11-16.

15. Ogungbamigbe TO, Ojurongbe OO, Ogunro PS, Olowe OA, Elemile PO. Prevalence and transmission pattern of Plasmodium falciparum infection in Osogbo metropolis, southwest, Nigeria. Afr J Med Med Sci. 2007 Dec; 36(4):305310

16. Prothero RM. Disease and mobility: a neglected factor in epidemiology Int J Epidemiol. 1977 Sep; 6(3):259-267.

17. Bruce-Chwatt LJ. Importation of disease. Yakugaku Zasshi 1968 Oct; 8(10):844.

18. Rajagopalan PK, Jambulingam P, Sabesan S, et al. Population movement and malaria persistence in Rameswaram Island. Soc Sci Med. 1986; 22(8):879-886.

19. Bates I, Fenton C, Gruber J, et al. Vulnerability to malaria, tuberculosis, and HIV/AIDS infection and disease. Part 1: determinants operating at individual and household level The Lancet Infectious Diseases. 2004; 4(5): 267-277.

20. Njoroge FK, Kimani VN, Ongore D, Akwale WS. Use of insecticide treated bed nets among pregnant women in Kilifi District, Kenya. East Afr Med J. 2009 Jul; 86(7):314-322.

21. Yakob L, Dunning R, Yan G . Indoor residual spray and insecticide-treated bednets for malaria control: theoretical synergisms and antagonisms. J R Soc Interface. 2010 Nov 17.

22. Tolhurst R, Nyonator F. Developing a methodology for the analysis of gender and malaria. Final Report on TDR Project 990984. Liverpool: Liverpool School of Tropical Medicine, 2002.

23.Grover-Kopec E, Kawano M, Klaver RW, Blumenthal B, Ceccato P, Connor SJ. An online operational rainfall-monitoring resource for epidemic malaria early warning systems in Africa. Malar J. 2005 Jan 21; 4:6.

24. Anastasia Phillips, Paul Bassett, Sebastian Zeki, Stanton Newman, Geoffrey Pasvol. Risk Factors for Severe Disease in Adults with Falciparum Malaria. Clinical Infectious Diseases 2009; 48:871_ 878.

25. Wong WC, Tam SM, Leung PW. Cross-border truck drivers in Hong Kong: their psychological health, sexual dysfunctions and sexual risk behaviours. J Travel Med.2007; 14(1), 20-30.

26. Chalwe V, Van geertruyden JP, Mukwamataba $\mathrm{D}$, et al. Increased risk for severe malaria in HIV1-infected adults, Zambia. Emerg Infect Dis. 2009 May; 15(5):749.

27. Jeremiah ZA, Jeremiah TA, Emelike FO. Frequencies of some human genetic markers and their association with Plasmodium falciparum malaria in the Niger Delta, Nigeria. J Vector Borne Dis 2010; 47(1):11-16.

28.Erhabor O, Adias TC, Hart ML. Effects of falciparum malaria on the indices of anaemia among pregnant women in the Niger Delta of Nigeria. Journal of ClinicalMedicine and Research 2010; 2(3):035-041.

29. Erhabor O, Jeremiah ZA, Adias TC, Hart ML. Thrombocytopenia in plasmodium parasitized pregnant women in the Niger Delta of Nigeria. Pathology and Laboratory Medicine International 2010:2 $1-5$.

30. Robert W. Snow, Carlos A. Guerra, Abdisalan M. Noor, Hla Y. Myint, Simon I. Hay. The global distribution of clinical episodes of Plasmodium falciparum malaria. Nature 2005; 434: 214-217. 